\title{
Analyzing Students' Cognitive Processing Patterns of A Socioscientific Issue Learning Activity with Online Discussion from A Process Perspective - Results of Quantitative Content Analysis and Lag Sequential Analysis
}

\author{
Huei-tse Hou*, Shu-ming Wang ${ }^{\dagger}$
}

\begin{abstract}
Socioscientific issues (SSI) are frequently used to enhance student's decision ability and critical thinking skills as SSI are generally ill-structured and lack of definite solutions. To help students be more immersed in the authentic problems, role-playing instructional strategy is commonly used. In this regard, this study thus employed a socioscientific issue role-playing learning activity, which students had to collaboratively work via asynchronous online discussion. While previous studies mostly focused on examining the collaboration outcomes, little attention has been paid to the collaboration process. Nonetheless, by inspecting learners' behavioral patterns in the collaboration process, the instructors would be able to provide adequate guidance for learners' can collaborate more efficiently. Therefore, this study seeks to propose a novel approach to depict the cognitive processing patterns that learners exhibited in the collaboration process by employing Quantitative Content Analysis (QCA) and Lag Sequential Analysis (LSA). Results of this study showed that students of different roles exhibited diverse cognitive processing patterns in online discussion. Results are discussed and expected to serve as guidance for conducting SSI related learning activity and subsequent collaborative learning research.
\end{abstract}

Keywords: socioscientific issue; role-playing; behavioral pattern analysis; sequential analysis

\section{Introduction}

Socioscientific issues (SSI) generally involve controversial scientific agendas, such as global climate change, alternative fuels, or genetic engineering. In addition, SSI are generally ill-structured, complicated and debatable as it involves diverse perspectives and lack of definite solutions [1]. In other words, it is controversial because varied opinions would be proposed subject to different perspectives, such as ethical, economical, or scientific. SSI promote students' decision ability as it requires students to think from diverse angle of views and propose arguments accordingly [2]. Students could learn better when situated in authentic problems. Thus,

\footnotetext{
* Graduate Institute of Applied Science and Technology, National Taiwan University of Science and Technology, Taipei, Taiwan

$\dagger$ Department of Information Management, Chinese Culture University, Taipei, Taiwan
} 
learning with SSI, which generally involved real world issues, can be an ideal approach to improve students' learning performance. Moreover, having students collaboratively work on SSI can help them learn better through cognitive interaction and knowledge co-construction among learner of diverse background and prior knowledge. Role-playing instructional strategy is commonly used to help students be immersed in learning. Adapting role-playing instructional strategy could help students be more focused and immersed into the problem to be solved with their assigned roles [3].

Previous studies have been investigated into how learners could benefit from socioscientific inquiry [4] and patterns of informal reasoning in the context of learning with socioscientific issues [5]. Most of the aforementioned SSI studies were conducted in traditional classroom. To explore students' learning performance, researchers generally used survey or interview to collect data [5]. However, survey might not properly reflect students' interaction patterns in the learning process while interview could require a lot of effort in collecting and analyzing data. Fortunately, with the advancement of information communication technology (ICT), nowadays, ICT-supported collaborative learning could enjoy the benefits such as eliminating temporal or spatial constraints of collaborative learning, easily integrating and sharing information of multiple sources in varied formats etc. Moreover, ICT can be used to collect the detailed data of students' interaction in the learning process automatically and arrange the data for further data analysis. Nonetheless, relatively little SSI-related research has been conducted in the context of ICT-supported learning. Besides, previous studies of collaborative learning mostly focused on examining the collaboration outcomes. However, by analyzing the collaborative process could improve the understanding of how learners collaborate from the process perspective [6].

To address these literature gaps and exploit the benefits of ICT-supported learning, this study employed a SSI learning activity with role-playing instructional strategy and used online discussion to support students' collaborative work. To depict the cognitive processing patterns exhibited in their online discussion, this study employed a mixed method approach that combined Quantitative Content Analysis (QCA) and Lag Sequential Analysis (LSA) to analyze students' discussions. This study seeks to apply a novel approach in analyzing the collaboration process and improve the understanding of students' cognitive processing pattern exhibited in a learning activity that involved SSI and role-playing instructional strategy in the context of ICT-supported learning. Results of this study are expected to be an exploration of learners' cognitive interaction and serve as guidance for orchestrating online SSI learning activity and subsequent collaborative learning research.

\section{Research Design}

\subsection{Participants \& Procedures}

Participants were 24 students enrolled in a Green Energy and Technology Introductory course of a university in northern Taiwan. To strengthen students' understanding of issues related to the nuclear power generation, a socioscientific issues learning activity with online asynchronous discussion was employed in the course. This study used online asynchronous discussion for following reasons. First of all, online asynchronous discussion is helpful to eliminate the temporal and spatial constraints as students could read and post message at anywhere and anytime [7]. Secondly, the temporal order and content of students' discussion can be automatically logged and arranged in the system for subsequent analysis. Thus, the content structure and behavioral patterns that students exhibited in online discussion could be analyzed using techniques such as 
Quantitative Content Analysis or Lag Sequential Analysis for better depicting learners' behavior in the learning process. In recent years, numerous studies have been employed these techniques to portray learners' behavioral patterns in ICT-supported learning [8][9].

The subject of discussion in this study is Nuclear power generation, which is a controversial issue around the world. While nuclear power generation is considered as an efficient and relatively low cost alternative, people are generally concerned about its safety issue and potential threat to the human beings and environment. Nuclear power generation could be an ideal subject for learning with SSI as it requires students to think from both the advantages and disadvantages of nuclear power generation. In this regard, this study designed a learning activity that incorporated a mock trial scenario. The mock trail involved local residents and a power company in northern Taiwan, where the nuclear power plant is located. In the scenario, the local residents file a suit against the power company for suspiciously causing the residents suffered from varied kinds of illness. Students were grouped into three 8-student groups. Each group was asked to play a role in the mock trial. The three different roles in the mock trial are prosecution group (local residents, who are against the nuclear power generation), defense group (the power company, who declare for the nuclear power generation), and general public, which served as observers and would discuss on the arguments proposed by the two other groups in the mock trial. This study included the general public group to explore the behavioral patterns of a group, which has lower level of involvement with the subject of discussion than the other two groups. In the learning activity, general public group was asked to discuss on the issues related to nuclear power generation. The description of the mock trial scene was provided to this group as well, only they didn't have to propose specific arguments.

The instructor gave students a detailed scenario description of the mock trial. Based on the roles they represented, students were asked to collaboratively collect related information, negotiate the meanings, summarize opinions, and propose their argumentations for the cross-examination session of the mock trial. Students were asked to discuss in a discussion board, which was specifically configured for the course in this study. The discussion board used in this study has general functions for students can discussion online, such as rich-text formatting, threaded subject format etc. Students' discussion was logged with time stamp for the purpose of data analysis. Students had a week for preparing the cross-examination of the mock trial. After a week of discussion, 545 messages in total were posted in the discussion board. In specific, the local residents group posted 235 messages, the power company group and the general public group both posted 155 messages respectively. This study thus retrieved all messages for subsequent analysis.

\subsection{Research methods}

To explore the behavioral patterns that students of different perspectives exhibited in an online SSI learning activity, this study employed Quantitative Content Analysis (QCA) and Lag Sequential Analysis (LSA) to analyze the retrieved messages [10][11].

All the messages in the discussion board were coded using a coding scheme before conducting LSA. In accordance with the purpose of this study, cognitive processing dimension of Revised Bloom's Taxonomy (RBT) was used [12]. RBT is a commonly used coding scheme to analyze learner's cognitive processing pattern by previous studies of similar context [8][13]. Using previously used coding scheme not only ensure the content validity, but also enable researchers to compare the results among similar contexts [11]. There are six cognitive processing phases in RBT, namely 'remember (denoted as B1 in this study)', 'understand (B2)', 'apply 
(B3)', 'analyze (B4)', 'evaluate (B5)', and 'create (B6)'. This study added one code to denote off-topic discussions (B7), such as casual social interaction, greeting etc. The description for each code is as shown in Table 1. To ensure the reliability of the coding results, two experienced coders, who are familiar with RBT, were invited to jointly code the messages. The Kappa coefficient was 0.85 , suggesting high inter-rater reliability [11]. Results of content analysis were compiled for further analysis. Results of QCA were delineated in section 3.1. To conduct LSA, this study thus arranged the coded messages in chronological order. LSA can be used to reveal the pattern and tendency of the behavior with statistical significance among behavioral sequences, or the order of the appearance of behaviors. LSA examines whether the appearance of one specific behavior followed by another specific behavior can reach statistical significance. In other words, LSA can used to determine whether an observed behavioral sequence is an outcome of random chance or not. LSA involves a series of matrix calculations [10]. In specific, three matrices are to be calculated, (1). sequential frequency transfer matrix; (2). condition probability matrix; (3). expected-value matrix. Next, the z-score of each behavioral sequence would be calculated based on the data in aforementioned matrices. Results would be arranged in an adjusted residuals table (Z-score table). The last step is to draw a sequential transfer diagram for illustrating the significant sequences using the data in the adjusted residuals table. Only those behavioral sequences with z-score higher than 1.96 was considered as significant $(p<0.05)$ and depicted in the sequential transfer diagram. The results of LSA were presented in section 3.2.

Table 1: Cognitive process dimension of RBT [12]

\begin{tabular}{|c|c|c|}
\hline Code & Process & Description \\
\hline B1 & Remember & $\begin{array}{l}\text { To access relevant knowledge form long-term } \\
\text { memory }\end{array}$ \\
\hline B2 & Understand & $\begin{array}{l}\text { To make sense of acquired knowledge; to associate } \\
\text { new knowledge with past experiences }\end{array}$ \\
\hline B3 & Apply & $\begin{array}{l}\text { To do a job or solve a problem through application } \\
\text { (procedures) }\end{array}$ \\
\hline B4 & Analyze & $\begin{array}{l}\text { To break down and analyze each component of } \\
\text { knowledge and note the relationship between the part } \\
\text { and the whole }\end{array}$ \\
\hline B5 & Evaluate & To judge and evaluate based on criteria and standards \\
\hline B6 & Create & $\begin{array}{l}\text { To piece different elements together and form a com- } \\
\text { plete and functional whole. To form a new structure by } \\
\text { re-assembling elements through the mental process }\end{array}$ \\
\hline B7 & Off-topic & $\begin{array}{l}\text { Discussions that are irrelevant to project topic, such as } \\
\text { social greeting or casual social interaction. }\end{array}$ \\
\hline
\end{tabular}

\section{Results and discussion}

\subsection{Results of QCA}

Table 2 showed the results of QCA. In this study, QCA was used to reveal the content structure of students' discussion. First of all, as shown in Table 2, the most cognitive process exhibited in students' discussions was 'understand (B2)'. This finding could be attributed to that the first of step of discussion or co-constructing knowledge is to understand the shared information. In this regard, students have to reason the meanings of the shared information and relate them to the 
subject of discussion. Secondly, a moderate portion of cognitive process of 'analyze (B4)' and 'create (B6)' were observed. As students had to propose arguments in the mock trial, they were expected to use the cognitive process of 'create' when proposing ideas in the discussions. In addition, before they can propose new ideas, students had to go over the details of the information, which requires the cognitive skills of analyzing. Third, we didn't observe much off-topic discussions. One possible explanation could be that students were assigned with clear roles. As they focused only playing the role, they would be more immersed to the problem and thus doing less casual social interaction, which was commonly seen in online discussions [14]. Lastly, in the results, we didn't observe cognitive process of 'apply (B3)' and 'evalute (B5)'. This finding could be attributed to the design of the learning activity. In other words, in the learning activity, the instructor didn't give students specific procedures or criteria for them to apply in evaluating the advantages, disadvantages, or potential risks of the nuclear power. Instead, the instructor gave students' clear descriptions of the scene, role groups and goals. Students were allowed to freely search and share information and then discuss on the collected information.

However, as shown in Table 2, three role groups showed similar content structure, or patterns, in their discussions. Thus, we might not be able to derive meaningful behavioral patterns unless we take into consideration the sequential patterns of cognitive process exhibited in the discussions. In this regard, this study further conducted the LSA. The following section described the results of LSA.

Table 2: Results of QCA

\begin{tabular}{lccccccc}
\hline Code & $\begin{array}{c}\text { Cognitive } \\
\text { Process }\end{array}$ & Local residents & Power Company & General Public \\
\hline \hline B1 & Remember & 0 & $0.00 \%$ & 0 & $0.00 \%$ & 0 & $0.00 \%$ \\
B2 & Understand & 168 & $71.49 \%$ & 115 & $74.19 \%$ & 105 & $67.74 \%$ \\
B3 & Apply & 0 & $0.00 \%$ & 0 & $0.00 \%$ & 0 & $0.00 \%$ \\
B4 & Analyze & 14 & $5.96 \%$ & 8 & $5.16 \%$ & 18 & $11.61 \%$ \\
B5 & Evaluate & 0 & $0.00 \%$ & 0 & $0.00 \%$ & 0 & $0.00 \%$ \\
B6 & Create & 37 & $15.74 \%$ & 23 & $14.84 \%$ & 21 & $13.55 \%$ \\
B7 & Off-topic & 16 & $6.81 \%$ & 9 & $5.81 \%$ & 11 & $7.10 \%$ \\
\hline Total & -- & 255 & $100 \%$ & 155 & $100.00 \%$ & 155 & $100.00 \%$ \\
\hline
\end{tabular}

\subsection{Results of LSA}

In this study, LSA was used to reveal the behavioral continuity and transition patterns of learners. Behavioral continuity refers to the statistically significant sequence that involves the same behavior. For example, B7 $\rightarrow$ B7 denotes a significant behavioral sequence, which an off-topic discussion was followed by another off-topic discussion. On the other hand, statistically significant behavioral sequences that involve two different behaviors are regarded as behavioral transition. For instance, B7 $\rightarrow$ B4 refers to an off-topic discussion were followed by a discussion that involved the 'analyze' cognitive process. Results of LSA were illustrated in Figure 1. For clarity, only statistically significant sequences were presented. 


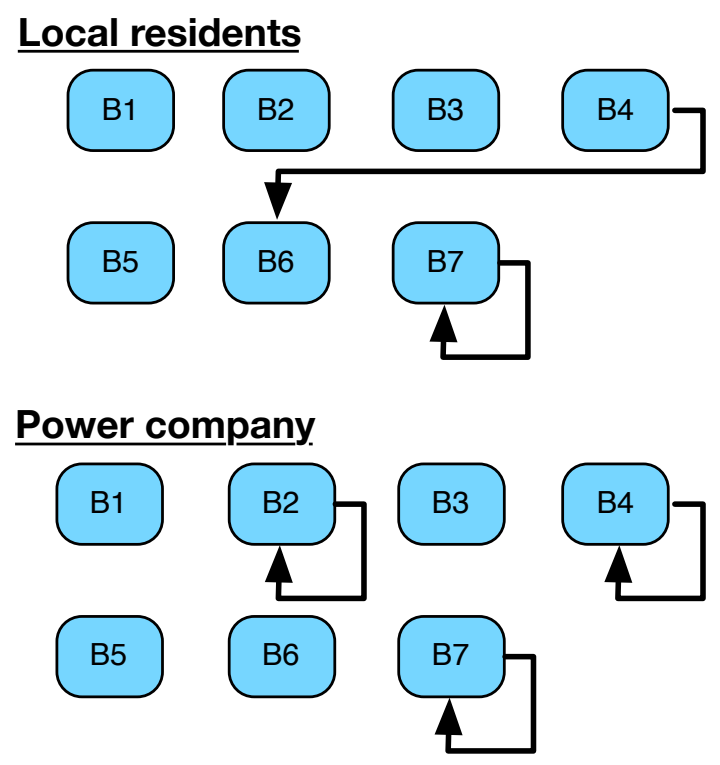

General Public

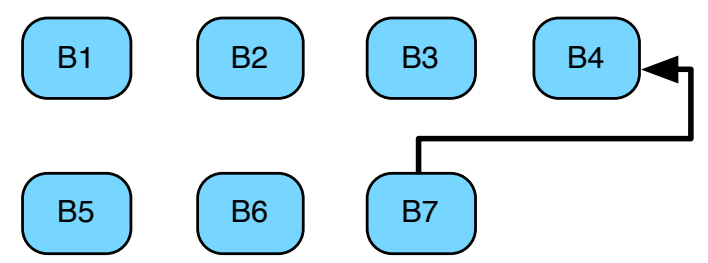

Figure 1: Results of the LSA

Results of LSA showed different cognitive processing patterns among different role groups. Firstly, the local residents group showed a behavioral transition from B4 (analyze) to B6 (create). This finding could be due to that the local residents group needed to analyze the collected evidence or information and then to propose arguments accordingly. Second, the group that played the power company role showed behavioral continuity for both B2 (understand) and B4 (analyze). These patterns showed that the discussions of power company group generally focused on reasoning and analyzing the information that commonly related to the nuclear power generation. This finding could be attributed to that the power company group had to properly respond to the plausible accusations that local residents would make. Meanwhile, both groups showed continuity in off-topic discussion (B7), such as social greeting or casual social interaction not related to the subject of discussion. Despite off-topic discussions were not related to the subject, they are not necessarily detrimental to online discussion. Previous studies pointed out that casual and frequent social interaction could be beneficial in promoting positive knowledge sharing climate [15], improving the extent of the group members' participation in discussion [16] and build emotional connections [17]. In other words, positive social interaction could be helpful in promoting group cohesion and encourage students to engage in the discussion. Regarding the general public group, the only significant behavioral pattern observed was a behavioral transition from B7 (off-topic) to B4 (analyze). This finding suggested that discussions of general public were mostly lack of focus in terms of behavioral continuity. Despite a significant portion of 'understand (B2)' was observed in the discussion of general public, there was no significant 
sequential pattern related to B2 in the LSA results. Moreover, a moderate portion of 'create (B6)' was observed. However, the LSA results suggested the general public group didn't focus on developing ideas or proposing arguments. One possible explanation of these findings could be the lower level of involvement to the subject of discussion of the general public than the other two groups. These findings also pointed out the importance of combining the results of QCA and LSA in order to derive meaningful behavioral patterns.

\section{Conclusion and future work}

One of the primary purposes of this study was to propose a novel approach, which can be used to explore the interaction patterns of students' collaboration process. This study tried to focus on analyzing the collaboration process rather than the collaboration outcomes. In this manner, this study thus designed an SSI learning activity with asynchronous online discussion to support students' collaboration. Students had to play different roles and propose argumentation accordingly. To delineate students' behavioral patterns in the collaboration process, QCA was used to depict the content structure of students' discussions while LSA was employed to reveal students' cognitive processing patterns that students exhibited in the online discussion. The approach that this study employed can be used to analyze the collaboration process for future research of similar context. In addition, results of this study could contribute to literature of SSI learning and was delineated and discussed as following.

First of all, our results showed varied behavioral patterns among the three role groups as each group represented a particular perspective of the SSI discussed in this study. As SSI generally involve complicated and debatable open-ended questions, these findings pointed out the advantages of adapting role-playing instructional strategy with SSI learning. Role-playing could help students be more immersed in the authentic problem and promote them to use diverse cognitive skills. Secondly, this study employed a SSI learning activity using asynchronous online discussion. Within a week, a total of 545 messages were posted on the discussion board by 24 students, suggesting students were generally willing to participate in the learning activity. Previous studies have pointed out the advantages of adapting online discussion with collaborative learning [18]. For instance, students would have more time to reflect on the subject before they post message. Students also can easily share online content and discuss accordingly. In addition, the detailed data of students would be logged in the system automatically for subsequent analysis. To exploit the benefits of ICT-supported learning, this study proposed a convenient approach for conducting online SSI learning activity and employed QCA and LSA to delineate students' interaction patterns in the learning process. Lastly, it is worth noting that simply using the results of content analysis to depict the behavioral patterns might lead to ambiguous interpretations. QCA results of this study showed similar patterns for the three role groups. It could be misleading if we drew a conclusion solely on the results of QCA. Nonetheless, combining the results of QCA and LSA, we would be more able to depict meaningful behavioral patterns exhibited in students' collaboration process. Future study are encouraged to adapt different socioscientific issues, such as global warming, alternative energies, or genetic engineering, and revise the learning activity design and analytic approach used in this study to explore learner's behavioral patterns. This approach would advance our understanding toward the interaction patterns of students' SSI inquiry with ICT-supported learning.

However, there were limitations that the subsequent phase of this study would seek to address to improve the understanding of learners' behavioral patterns in learning activities involving SSI. First of all, the discussion time given to students could be too short. In this study, students had 
one week to discuss and prepare their propositions for the cross-examination session of the mock trial. However, one week might not be ample enough for students to dig into the subject of discussion deeply and employ diverse cognitive skills [14]. By giving students more time to reflect on the project, we could expect to observe a more diversified patterned and advanced cognitive processes in their discussions. Secondly, this study used the cognitive process dimension of Revised Bloom's Taxonomy to denote the cognitive process patterns. This approach would limit our understanding of students' collaboration patterns as the coding scheme only represented single perspective. There were other coding schemes, which were commonly used to analyze learners' online discussions, such as Interaction analysis model (IAM), which was commonly used to depict students' social knowledge construction behaviors [19]. Future study can employ the analytic approach of this study and incorporate diverse perspectives to explore learners' behavioral patterns in the online discussion. In this manner, researchers would be able to build a more comprehensive understanding of learners' collaborative behavioral patterns in online discussion. Lastly, in this study, we invited two experienced coders to code the retrieved message according the coding scheme. This approach requires quite a bit of time and effort if there is a substantial number of messages retrieved. With the advancement of technology, future research could use techniques such as text mining or sentimental analysis to help researcher processing the messages. Even more, future research could build a collaboration support system that summarizes the key patterns and status of the learning activity on the fly to help instructors to provide adequate guidance to the learning activity in a timely fashion.

\section{Acknowledgement}

This research was supported by the projects from the Ministry of Science and Technology, Reublic of China, under contract number MOST-103-2511-S-034-001, MOST-104-2511-S-034-002, MOST-102-2511-S-011-001-MY3 and MOST-100-2628-S-011-001-MY4

\section{References}

[1] T. D. Sadler and D. L. Zeidler, 'Patterns of Informal Reasoning in The Context of Socioscientific Decision Making,' Journal of Research in Science Teaching, vol. 42, no. 1, 2005, pp. 112.

[2] T. D. Sadler and D. L. Zeidler, 'The Significance of Content Knowledge for Informal Reasoning Regarding Socioscientific Issues: Applying Genetics Knowledge to Genetic Engineering Issues,' Science Education, vol. 89, no. 1, 2005, pp. 71.

[3] L. D. Chen, M. N. Frolick, and A. Muthitacharoen, 'Investigating The Use of Role Play Training to Improve The Communication Skills of IS Professionals: Some Empirical Evidence,' Journal of Computer Information Systems, vol. 43, no. 3, 2003, pp. 67.

[4] T. D. Sadler, S. Barab, and B. Scott, 'What Do Students Gain by Engaging in Socioscientific Inquiry?,' Research in Science Education, vol. 37, no. 4, 2007, pp. 371.

[5] T. D. Sadler, 'Informal Reasoning Regarding Socioscientific Issues: A Critical Review of Research,' Journal of Research in Science Teaching, vol. 41, no. 5, 2004, pp. 513. 
[6] Dillenbourg, P., Järvelä, S., \& Fischer, F. (2009). The Evolution of Research on Computer-Supported Collaborative Learning. In N. Balacheff, S. Ludvigsen, T. de Jong, A. Lazonder, \& S. Barnes (Eds.), Technology-Enhanced Learning (pp. 3-19): Springer Netherlands.

[7] A. P. Rovai, 'Facilitating Online Discussions Effectively," The Internet and Higher Education, vol. 10, no. 1, 2007, pp. 77.

[8] H. T. Hou, S. M. Wang, P. C. Lin, and K. E. Chang, 'Exploring The Learner'S Knowledge Construction and Cognitive Patterns of Different Asynchronous Platforms: Comparison of An Online Discussion Forum and Facebook,' Innovations in Education and Teaching International, 2013, pp. 1.

[9] A. C. Jeong, 'The Sequential Analysis of Group Interaction and Critical Thinking in Online Threaded Discussions,' The American Journal of Distance Education, vol. 17, no. 1, 2003, pp. 25.

[10] R. Bakeman and J. M. Gottman, Observing Interaction : An Introduction to Sequential Analysis, 2nd ed. New York: Cambridge University Press, 1997.

[11] L. Rourke and T. Anderson, "Validity in Quantitative Content Analysis," Educational Technology Research \& Development, vol. 52, no. 1, 2004, pp. 5.

[12] L. W. Anderson, D. R. Krathwohl, and B. S. Bloom, A Taxonomy for Learning, Teaching, and Assessing : A Revision of Bloom's Taxonomy of Educational Objectives, Complete ed. New York: Longman, 2001.

[13] H. T. Hou, 'Analyzing the Learning Process of an Online Role-Playing Discussion Activity,' Journal Educational Technology \& Society, vol. 15, no. 1, 2012, pp. 211.

[14 ]Lin, P.-C., Hou, H.-T., Wang, S.-M., \& Chang, K.-E., “Analyzing Knowledge Dimensions and Cognitive Process of A Project-Based Online Discussion Instructional Activity Using Facebook in An Adult and Continuing Education Course.," Computers \& Education, vol. 60, no. 1, 2013, pp. 110.

[15] G. W. Bock, R. W. Zmud, K. Young-Gul and L. Jae-Nam, 'Behavioral Intention Formation in Knowledge Sharing: Examining The Roles of Extrinsic Motivators, Social-Psychological Forces, and Organizational Climate,' MIS Quarterly, vol. 29, no. 1, 2005, pp. 87.

[16] K. Kreijns, P. A. Kirschner, W. Jochems and H. Van Buuren, 'Determining Sociability, Social Space, and Social Presence in Asynchronous Collaborative Groups,' CyberPsychology \& Behavior, vol. 7, no. 2, 2004, pp. 155.

[17] G. Veletsianos, 'How Do Learners Respond to Pedagogical Agents That Deliver Social-Oriented Non-Task Messages? Impact On Student Learning, Perceptions, and Experiences,' Computers in Human Behavior, vol. 28, no. 1, 2012, pp. 275.

[18] Lucas, M., Gunawardena, C., \& Moreira, A., “Assessing Social Construction of Knowledge Online: A Critique of The Interaction Analysis Model," Computers in Human Behavior, Vol. 30, 2014, pp. 574. 
[19] C. N. Gunawardena, C. A. Lowe, and T. Anderson, 'Analysis of A Global Online Debate and The Development of An Interaction Analysis Model For Examining Social Construction of Knowledge in Computer Conferencing," Journal of Educational Computing Research, vol. 17, no. 4, 1997, pp. 397. 